\title{
Erratum to: Can the chemotherapeutic agents perform anticancer activity through miRNA expression regulation? Proposing a new hypothesis
}

\author{
Chiranjib Chakraborty $^{1}$ C. George Priya Doss ${ }^{2} \cdot$ Renu Sarin $^{3} \cdot$ Minna J. Hsu $^{4}$. \\ Govindasamy Agoramoorthy ${ }^{5}$
}

Published online: 1 April 2015

(C) Springer-Verlag Wien 2015

\section{Erratum to: Protoplasma}

DOI 10.1007/s00709-015-0776-7

The original publication of this article contains an error.

The word "though" that appeared in the article title should be corrected as "through". The correct title is "Can the chemotherapeutic agents perform anticancer activity through miRNA expression regulation? - Proposing a new hypothesis".

The online version of the original article can be found at http://dx.doi.org/ 10.1007/s00709-015-0776-7.

Chiranjib Chakraborty

drchiranjib@yahoo.com

$\triangle$ Minna J. Hsu

hsumin@mail.nsysu.edu.tw

1 Department of Bio-informatics, School of Computer and Information Sciences, Galgotias University, Greater Noida, Uttar Pradesh, India

2 Medical Biotechnology Division, School of Biosciences and Technology, VIT University, Vellore, India

3 Department of Chemistry, Galgotias College of Engineering and Technology, Greater Noida, Uttar Pradesh, India

4 Department of Biological Sciences, National Sun Yat-sen University, Kaohsiung, Taiwan

5 College of Pharmacy and Health Care, Tajen University, Yanpu, Pingtung 907, Taiwan 